Ondřej PEŠEK

Université de Bohême du Sud, České Budějovice

\title{
LES ASTRES ET LA MORT - ETUDE DE LA STRUCTURATION D'UNE SEQUENCE TEXTUELLE DU ROMAN DE LA ROSE
}

\section{Introduction}

Comme son titre le suggère, l'étude que nous présentons ici relève de la linguistique textuelle. Consacrée à un court passage du Roman de la Rose de Jean de Meun, elle est conçue comme une application des méthodes modernes de l'analyse textuelle au matériel linguistique de l'ancien français. Avant de présenter les analyses concrètes, il nous paraît nécessaire d'expliciter les raisons qui nous ont amené à choisir ce texte et de préciser, ne serait-ce que très brièvement, les bases théoriques de notre méthodologie.

1.1. Par son envergure et par sa complexité, le Roman de la Rose de Jean de Meun se distingue nettement parmi les œuvres «savantes » du Moyen Âge. Sur quelques 17000 vers sont traitées des questions non seulement scientifiques (astronomie, optique, alchimie), mais aussi politiques, morales et sociales. Il s'agit d'une véritable somme de connaissances qui reflètent les préoccupations et les discours propres à la couche sociale des clercs médiévaux. La variété des matières exposées va de pair avec la variété compositionnelle et typologique: Jean de Meun raconte des histoires, décrit une multitude d'objets divers, explique les principes des choses, argumente en faveur de ses convictions, met en scène des dialogues, bref exploite d'une manière riche et ingénieuse toutes les modalités de la typologie séquentielle. $\mathrm{Vu}$ sous cet angle, son poème est particulièrement intéressant pour l'analyse textuelle des discours.

1.2. C'est dans ce cadre théorique que s'inscrit notre étude. Elle s'inspire notamment de la théorie des séquences textuelles développée par Jean-Michel ADAM $(1994,1999,2005)$; par conséquent, les termes techniques qui apparaissent dans notre analyse sont employés conformément aux travaux de ce dernier. Les ouvrages de Jean-Michel Adam ne représentent pas pour autant la seule source dans lesquelles nous puisons : à bien des égards, les analyses que nous avançons s'inspirent aussi des travaux d'Oswald Ducrot, de ses collaborateurs et disciples (nottament J. Moeschler).

Avant de commencer l'analyse séquentielle de notre extrait, rappelons brièvement ce que J.-M. Adam entend par le terme séquence et période textuelles. Il s'agit des macro-unités textuelles formées par le liage des propositions-énoncés. La différence entre une séquence et une période est plutôt graduelle - cette dernière est moins longue et plus faiblement typée que la séquence. Les séquences sont, quant à elles, profondément typées. Ce sont des entités autonomes, structurées et décomposables en parties. J.-M. Adam distingue cinq types de séquences - narrative, descriptive, argumentative, explicative et dialogale - qui correspondent aux opérations cognitives et pragmatiques fondamentales. Pour chacune des cinq séquences J.-M. Adam propose un schéma prototypique, qui, dans le discours, ne s'actualise pas toujours sous cette forme puisque certaines des propositions constitutives du schéma peuvent ne pas être explicitées dans le texte. 
Ce schéma est donc le prototype de la catégorie, son meilleur représentant. On voit bien que la linguistique textuelle s'inspire de la sémantique, qui a introduit dès les années 70 la notion de prototype afin de rendre compte des mécanismes cognitifs de la catégorisation des objets du monde par l'intermédiaire de la langue (les schémas prototypiques sont pour chacune des séquences la même chose que le moineau l'est pour la catégorie des oiseaux). Les combinaisons des séquences déterminent la structure d'un tout textuel. Les différentes séquences peuvent être soit identiques (on a affaire à un texte homogène du point de vue séquentiel) soit différentes (textes hétérogènes). J.-M. ADAM (2005 : 184) distingue trois types de base d'agencement des séquences : succession des séquences, enchâssement des séquences et montage en parallèle.

1.3. Portant sur un court extrait du Roman de la Rose, notre étude n'exploite que très modestement les possibilités qu'offre aux linguistes le texte de Jean de Meun. Nous n'avons pas l'ambition de rendre compte de la complexité et de l'hétérogénéité séquentielle du Roman. Nous avons préféré, dans un premier temps, analyser seulement une seule séquence, en vue de montrer quelles peuvent êtres les modalités concrètes d'actualisation d'un type donné d'une séquence textuelle. L'examen détaillé de son agencement nous permettra de formuler quelques hypothèses relatives aux propriétés logico-pragmatiques de certaines structures textuelles constitutives de la séquence analysée. Ce faisant, nous prêterons une attention particulière aux rôles des différents connecteurs et marqueurs de prise en charge énonciative qui figurent dans le passage analysé.

\section{Le texte}

Notre passage représente l'une des nombreuses digressions dont Jean de Meun, s'éloignant de la trame d'action du Roman, profite pour exposer la matière savante. Il fait partie de la confession de Nature qui s'étend sur quelques 3000 vers. Agenouillée devant Genius, son prêtre, Nature passe en revue l'univers tout entier : les corps célestes, les éléments, les plantes, les animaux, afin de pouvoir à la fin de sa confession formuler sa plainte contre l'homme, la seule créature qui n'obéit pas à ses règles. La dissertation sur les corps célestes est l'occasion pour Jean de Meun d'introduire dans son texte des réflexions philosophiques relatives au prédéterminisme astrologique, à la prescience divine, à la prédestination et au libre arbitre. Le passage que nous allons analyser est consacré au prédéterminisme astrologique : en évoquant la mort d'Empédocle et le sort d'Origène, Jean de Meun pose la question de savoir dans quelle mesure leur destin a été prédéterminé par les astres et donc était inévitable. Voici le texte du passage ${ }^{1}$; il s'agit des vers 17063 - 17104 (édition d'Armand Strubel) :

(a) Si dit on (b) que les destinees / Leur orent tels morz destinees... /

(c) Des lors qu'il ... pristrent leur nacions / En teles constellacions; /

\footnotetext{
${ }^{1}$ Nous avons abrégé un peu le texte original en supprimant les vers qui allaient dans le même sens et qui ne faisaient que redoubler ou gloser les vers que nous avons gardés. Les connecteurs dont le fonctionnement sera analysé dans cette étude sont en majuscules et en caractères gras. Les propositions constitutives de la séquence sont marquées par une lettre minuscule entre parenthèses. Les marqueurs de prise en charge énonciative sont en italiques.
} 
(d) Et par droite necessité... / Leur couvient tel mort recevoir. / (e) MAIS $_{1}$ je sai bien, trestout de voir, / (f) Conbien que li ciel i travaillent... / (g) Qui les enclinent a ce faire... / Par la matire obeïssant / Qui leur cuers va si flechissant, / (h) Si pueent il bien par doctrine... / Et par bonté d'entendement, / Procurer qu'il soit autrement... / (i) $\mathbf{C A R} \mathbf{1}$ quant de sa propre nature / Contre bien et contre droiture / Se veult homme ou fame atorner, / (j) Raisons l'en puet bien destorner, / (k) Pour qu'il la croie seulement. / (l) LORS ira la chose autrement, / (m) $\mathbf{C A R} \mathbf{R}_{\mathbf{2}}$ autrement puet il bien estre, / (n) Que que facent li cors celestre / (o) Qui mout ont grant pooir, SANZ FAILLE... / (p) MAIS $\mathbf{2}_{\mathbf{2}}$ n'ont pooir contre reson, / (q) $\mathbf{C A R}_{\mathbf{3}}$ bien set chascuns sages hom, / (r) Qu'il ne sont pas de raison maistre / (s) N'il ne la firent mie nestre.

\section{Analyse séquentielle}

3.1. Nous proposons d'analyser ce passage (propositions-énoncés a-s) comme l'actualisation d'une séquence argumentative. Pour rendre compte de la composition de cette séquence et pour pouvoir décrire d'une manière cohérente l'agencement des propositions-énoncés constitutives, nous allons référer l'extrait analysé au schéma prototypique de la séquence argumentative, proposé par J.-M. ADAM (2005: 159) $)^{2}$. Le prototype de la séquence argumentative selon J.-M. Adam est structuré de la manière suivante :

\begin{tabular}{|c|c|c|c|c|}
\hline Thèse antérieure & + Données & ----------------- & Donc probablement & --------- Conclusion \\
\hline P. $\arg 0$ & P. $\arg 1$ & $\begin{array}{c}\uparrow \\
\text { Étayage (puisque) } \\
\text { P. arg } 2 \\
\text { (principes, bases) }\end{array}$ & $\begin{array}{c}\uparrow \\
\text { à moins que } \\
\text { P. arg } 4 \\
\text { (restriction) }\end{array}$ & $\begin{array}{r}\text { (nouvelle thèse) } \\
\text { (P. } \arg 3)\end{array}$ \\
\hline
\end{tabular}

Précisons dès le début que la séquence argumentative que représente notre texte n'est pas actualisée sous cette forme prototypique idéale. En effet seules les P. $\arg 0$ et P. arg 1 sont explicitées : P. arg 0 correspondant aux énoncés (a, b, c, d), P. arg 1 aux énoncés (e, f, g, h). De plus, le texte comporte des propositions qui semblent ne pas entrer dans le schéma : le reste du texte, propositons-énoncés (i-s). Dans les lignes qui suivent nous effectuerons une analyse détaillée de chacune des parties constitutives de ce texte afin de démontrer quel est leur statut dans le cadre de la séquence. Cette analyse nous permettra de faire ressortir les spécificités de cette séquence non prototypique et d'en tirer des implications d'ordre général.

\subsection{Analyse de la $P$. $\arg 0$}

Dans la terminologie de J.-M. Adam, la P. arg 0 représente la thèse antérieure qui est à réfuter à l'aide des arguments présentés dans la $\mathrm{P}$. arg 1 . Comme nous l'avons vu, la P. arg 0 est elle-même constituée de plusieurs propositions-énoncés (a, b, c, d). Même si elles ne comportent pas de marques explicites de leur visée argumentative ni de leur articulation, le mouvement argumentatif qui résulte de

\footnotetext{
${ }^{2}$ L'abréviation P. arg signifie macro-proposition argumentative. Pour des illustrations concrètes du prototype de la séquence argumentative, nous renvoyons le lecteur à J.-M.ADAM (1994 : 101-126) et $(2005,157-162)$
} 
leur sens correspond au schéma de base d'une structure textuelle argumentative (voir le schéma ci-dessus) à partir de laquelle J.-M. ADAM (2005 : 158) dérive le prototype séquentiel. Il est important de faire remarquer que la validité de l'argumentation de P. arg 0 de notre passage est placée sous l'autorité de si dit on, c'est-à-dire celle d'un point de vue $(\mathrm{PdV})$ qui n'est pas celui du locuteur.

Si dit on que [Données (D0)-----------------doncConclusion $(\mathrm{C} 0)]$

Étayage (puisque Ét0)

Les différentes parties constitutives de ce schéma correspondent aux énoncés suivants de l'extrait du Roman de la Rose :

$\mathrm{D} 0=\mathrm{b}+\mathrm{c}$

$\mathrm{C} 0=\mathrm{d}$

Ét0 implicite

La période argumentative que l'on obtient ainsi peut être résumée comme suit :

D0 ----donc---- $\quad \mathrm{C} 0$

(Les astres ont déterminé telle mort de ces gens) （cette mort est inévitable)

Le passage de D0 à C0 est possible grâce à l'application de l'inférence qui étaye le raisonnement (d'où le terme «étayage »). Le principe de l'inférence dans l'argumentation est défini par J.-M. ADAM (1994: 105) ainsi : « un principe général qui évite qu'on soit obligé d'introduire d'autres données et vient jeter, en quelque sorte, le pont entre donnée et conclusion». Très souvent, il s'agit du passage de la classe à un membre de la classe. Le principe de l'inférence décrit par J.-M. Adam est analogue au principe que J. MOESCHLER (1985: 68) appelle «topos » et qu'il définit ainsi : «règle générale qui rend possible une argumentation particulière ». Un topos, selon J. Moeschler, n'est pas une règle de la logique, il s'agit d'une règle communément admise, mais qui peut être contesté ou réfuté.

Dans notre cas, le topos qui sous-tend l'argumentation de la P Arg 0 est la relation d'implication entre le déterminisme astral et la réalisation de ce qui est déterminé, ce que l'on pourrait paraphraser comme suit :

T0 : Si une chose est déterminée par les astres, elle est inévitable.

En appliquant ce topos, on passe alors du général - une chose - au particulier une mort concrète. Le schéma complet de la période est le suivant : 


\section{D0 ----donc---- C0 \\ (Les astres ont déterminé telle mort de ces gens) ～(cette mort est inévitable) \\ puisque Ét0 (implicitement admis) \\ (T0 : si une chose est déterminée par les astres, elle est inévitable)}

\subsection{Le noyau de la séquence}

Comme on l'a dit plus haut, cette période est enchâssée dans la séquence argumentative actualisée dans notre extrait comme P. Arg 0. Cette séquence, telle qu'elle apparaît dans le texte de Jean de Meun, peut être schématisée de la façon suivante :

«On dit que D0 donc C0, MAIS je sais que même si D0 dans une grande mesure, pas nécessairement $\mathrm{C} 0$.»

Nous sommes persuadé que dans l'argumentation, cette structure sert à faire ressortir la non-pertinence du topos qui relie causalement D0 à C0. Cela est possible parce qu'en énonçant on dit que, le locuteur n'est pas le garant de la validité des propos avancés qui sont ainsi situés en dehors de son univers de croyance. En nous basant sur la description classique du MAIS cité par exemple dans O. DUCROT (1980: 97) nous pouvons paraphraser cette structure comme suit : «Vous pourriez être ainsi amenés à admettre la validité de l'argumentation 'D0 donc C0' comme beaucoup le font (on dit), mais il ne faut pas, car elle n'est pas nécessairement valide. » Le marqueur de prise en charge énonciative ${ }^{3}$ je sais,

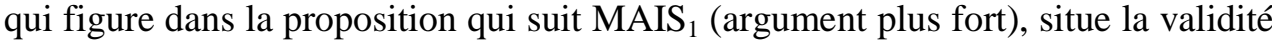
des propos sous la responsabilité du locuteur, ce qui leur donne, dans l'argumentation, plus de poids persuasif. Le rôle des marqueurs de prise en charge énonciative qui figurent dans cette structure est particulièrement important, nous y reviendrons dans la dernière partie de cette étude. Comme nous l'avons postulé, la conclusion qui résulte de cette structure argumentative est la réfutation de la pertinence du topos $\mathrm{T} 0$ qui fonde l'argumentation $\mathrm{D} 0 \rightarrow \mathrm{C} 0$. On arrive à cette conclusion par un mouvement de pensée basé sur l'actualisation dans la P. $\arg 2$ du topos T2 suivant :

T2: S'il n'est pas vrai que [DO donc CO], le topos T0 fondant $\mathrm{D} 0 \rightarrow C \mathrm{CO}$ n'est pas pertinent.

Appliquons maintenant ce qui vient d'être démontré au schéma général de la séquence argumentative cité plus haut tout en indiquant les énoncés concrets du passage du Roman de la Rose qui s'inscrivent dans les différents composants de ce schéma :

\footnotetext{
${ }^{3}$ Terme utilisé par J.-M. ADAM (2005 : 117). Ces morphèmes ont pour rôle d'attribuer une portion de texte à un point de vue $(\mathrm{PdV})$.
} 
Thèse ant. (P. arg 0)

(a) si dit on que $[\mathrm{D} 0(\mathrm{~b}+\mathrm{c})$ donc $\mathrm{C} 0(\mathrm{~d})$, fondé sur le topos $\mathrm{T} 0]$

$+$

MAIS $_{1}$ Données (P. arg. 1)

(e) je sai que [combien que $\mathrm{D} 0(\mathrm{f}+\mathrm{g})$ pas nécessairement $\mathrm{C} 0(\mathrm{~h})]$

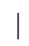

(actualisation du topos T2 : s'il n'est pas vrai que [DO donc CO], le topos $T 0$ fondant $D O \rightarrow C O$ n'est pas pertinent)

।

donc

$\downarrow$

Conclusion C (P. arg 3) implicite :

le principe « si une chose est déterminée par les astres, elle est inévitable » n'est pas pertinent

La thèse du noyau de la séquence argumentative (propositions-énoncés a-h) pourrait être résumée ainsi :

«On dit que si les astres ont déterminé la mort d'un homme, il ne peut pas l'éviter, mais je sais que quoique les astres exercent une grande influence sur l'homme, il peut éviter cette mort. L'homme est donc indépendant par rapport au déterminisme astrologique. »

Les énoncés (a-h) articulés par MAIS $_{1}$ représentent le noyau de la séquence. Regardons maintenant quel est le statut des autres propositions de notre passage par rapport à la structure nucléaire.

\subsection{La suite de la séquence, les connecteurs et leur rôle argumentatif}

Les propositions (i) et (j) qui suivent, introduites par $\mathrm{CAR}_{1}$ présentent une justification ${ }^{4}$ de l'énonciation de l'argument fort $(\mathrm{h})$ : on peut asserter que le pouvoir des corps célestes sur le destin des hommes n'est pas absolu car l'homme est doué de la raison qui peut s'opposer aux inclinations dues à l'action des astres. La proposition (l) introduite par LORS revêt ici une valeur consécutive qui enchaîne avec la réserve émise en (k).

Le l'ensemble des propositions qui suit, introduit par $\mathrm{CAR}_{2}$, est à considérer comme une justification à la fois de l'argument (h) et du (l) issu du raisonnement $[(\mathrm{i}+\mathrm{j}+\mathrm{k}) \rightarrow \mathrm{l}]$ et reformule en quelque sorte la thèse de ce passage - grâce à la raison, les choses peuvent se passer autrement que ne le veulent les corps célestes. Sous la portée de ce $\mathrm{CAR}_{2}$ se trouve la période argumentative SANZ FAILLE (o),

${ }^{4}$ cf. à ce sujet $\lambda-1(1975: 265)$ : «Les énoncés du type $p$ car $q$ servent, en règle générale, à accomplir deux actes de langage successifs. Le premier consiste à énoncer $p$, et le second à fournir une justification du premier : l'énonciation de $q$ se présente donc comme destinée à légitimer celle de p.» 
$\mathrm{MAIS}_{2}$ (p) dont la conclusion est ainsi au service de la justification. Cette période se prête à l'analyse tout à fait classique du MAIS argumentatif :

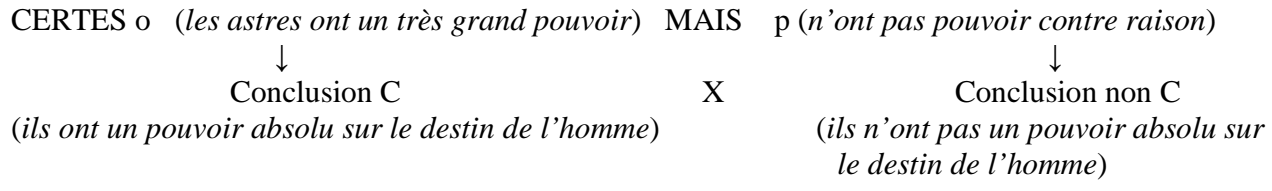

Conclusion C

(ils ont un pouvoir absolu sur le destin de l'homme)

$\mathrm{X}$

(ils n'ont pas un pouvoir absolu sur le destin de l'homme)

$\mathrm{CAR}_{3}$ qui introduit les propositions (q-s) justifie le fait que la Nature asserte la proposition (p) à la place de l'argument plus fort, qui dans le cadre de la période

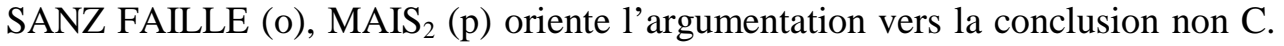
$\mathrm{CAR}_{3}$ fonctionne ici d'une manière exemplaire et illustre bien ce que dit à propos de ce connecteur A. DELBEY $(1988,410-411)$ : la proposition introduite par CAR $\mathrm{C}_{3}$ fait forcément partie de l'univers de croyance du locuteur, en l'énonçant, le locuteur en asserte la vérité. Mais cette vérité peut être prise en charge non seulement par le locuteur seul, mais peut être partagée par un espace de croyance plus vaste, même universel, auquel le locuteur adhère naturellement. Si c'est le cas, une référence explicite à cet espace de croyance figure dans l'énoncé. Le marqueur bien set chascuns sages hom en est une parfaite illustration.

\subsection{Structuration séquentielle}

Nous venons d'expliciter le mouvement argumentatif de notre extrait « orchestré » par les différents connecteurs. Il est maintenant nécessaire d'analyser les rapports respectifs entre les différents ensembles de propositions-énoncés (séquences/périodes) afin de pouvoir déterminer la structuration séquentielle du passage en question. Nous nous devons donc de répondre à la question de savoir si nous avons affaire à un texte homogène ou hétérogène du point de vue séquentiel, quel est le type d'agencement des séquences, si celles-ci sont hiérarchiquement équivalentes ou si au contraire ou peut y reconnaître une dominante.

Puisque le texte contient plusieurs occurrences de connecteurs de nature différente (MAIS et CAR) nous pourrions être amené à postuler qu'il s'agit d'un texte hétérogène du point de vue séquentiel. Il ne fait pas de doute que MAIS est un connecteur qui signale d'une manière claire et évidente la présence d'une période/séquence argumentative, mais qu'en est-il de CAR ? Dans la mesure où il peut, dans certains cas, commuter avec parce que (cf. O. SOUTET 162), on serait tenté de ranger les ensembles de propositions qui sont sous la portée du CAR dans le domaine des périodes/séquences explicatives. Nous sommes toutefois persuadé que le fonctionnement du CAR au niveau des séquences textuelles de notre extrait doit être interprété différemment. Dans les périodes argumentatives du type $p$ MAIS q CAR $r$, qui est actualisé à trois reprises dans le texte analysé, $r$ est toujours une justification de l'énonciation de l'argument le plus fort (voir ci-dessus) et en soutenant la validité de $q$, il renforce le mouvement argumentatif de la période vers la conclusion $q \rightarrow C$ ainsi que MAIS l'indique. Étant donné que « ...un énoncé $p$ car $q$ se donne comme dirigé vers $p$, et non pas vers $q(\lambda-1,1975: 270)$ », la proposition $r$ de la structure $p$ MAIS $q$ CAR $r$ est entièrement au service de 
l'argumentation, CAR ne signalant pas l'ouverture d'une autre séquence différente. Même si CAR peut introduire une explication, celle-ci sert toujours à la justification du $q$, car «le plus souvent, la meilleure manière de justifier l'énonciation assertive de $q$ consiste à indiquer la cause (ou une des causes) du fait asserté en $q$. Justifier et expliquer sont alors une seule et même chose (O. SOUTET, 1992 : 162) ». Contrairement aux enjeux des séquences explicatives, le but de la (des) proposition(s) qui est (sont) sous la portée du CAR n'est pas de donner à

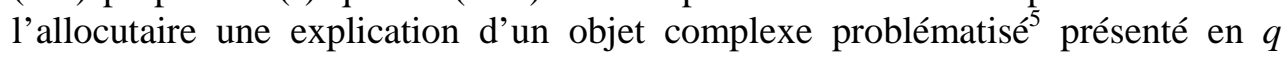
(«voilà pourquoi $q$ »), mais de justifier l'assertion d'un argument. C'est pour toutes ces raisons que nous considérons les propositions-énoncés de notre extrait introduites par CAR comme faisant partie intégrante de la séquence argumentative.

Le fonctionnement argumentatif de la structure du type $p$ MAIS $q$ CAR $r$, actualisée à trois reprises dans cet extrait, nous semble très intéressant, à tel point qu'il mérite que nous lui consacrions une analyse un peu plus détaillée. Notre analyse va entre autres confirmer ce que nous avons dit à propos du statut qu'ont les propositions-énoncées introduites par CAR dans le cadre des séquences textuelles.

\section{Les structures du type $p$ [MAIS $q C A R r]^{6}$}

Il nous semble en effet qu'il est possible de distinguer deux types différents de la structure $p$ [MAIS $q C A R r$ ], chacune pouvant être divisée en deux sous-types en fonction du caractère du MAIS articulant les propositions $p$, $q$. Nous allons sortir un instant du cadre du Roman de la Rose: pour tester les possibilités d'actualisation de la structure $p$ [MAIS q CAR $r$, nous allons considérer les argumentations dans une situation fictive, imaginée pour notre propos. Cette petite digression nous permettra de vérifier notre hypothèse de départ.

La situation fictive est la suivante : Une ambassade A doit envoyer en Chine un agent dont la tâche serait de persuader Mme Y de favoriser le pays A lors de la distribution des commandes d'État. On sait que Mme Y aime bien les hommes et apprécie ceux qui parlent chinois. Les diplomates de l'ambassade A délibèrent pour décider si l'agent $\mathrm{X}$ est la bonne personne qu'il faudrait envoyer pour effectuer cette mission.

\subsection{Premier type}

Le premier cas de figure de la structure $p$ [MAIS q CAR $r$ ] s'actualise dans le dialogue suivant :

Conseiller $1:$ X est moche ! »

Conseiller 2 : «X est moche, mais il faut l'envoyer à la mission, car il a du succès auprès des femmes !»

Conseiller 3 : «X est moche, mais il faut l'envoyer à la mission, car il parle très bien chinois !»

\footnotetext{
${ }^{5}$ cf. J.-M. ADAM (2005: 166)

${ }^{6}$ Nous préférons utiliser les crochets $p$ [MAIS $q$ CAR $r$ ] pour marquer que la proposition $r$ est du point de vue argumentatif et textuel intimement liée à la proposition $q$.
} 
Dans ce cas la proposition $q$ est en effet l'explicitation de la conclusion vers laquelle le locuteur (en l'occurrence les Conseillers 2 et 3 ) oriente son argumentation et qui est le contraire de la conclusion qui découle de la proposition $p$ (en l'occurrence, la conclusion en faveur de laquelle argumente le Conseiller 1 en disant «X est moche »). La proposition $r$ est alors une justification de l'énonciation de cette conclusion, l'argument dont il faudrait tirer la conclusion énoncée en $q$ si elle n'était pas explicitée. La différence entre les interventions des conseillers 2 et 3 , qui argumentent en faveur de la même conclusion, consiste en la nature différente des propositions $r$ respectives. Dans le premier cas $r$ est contraire à l'implication ${ }^{7} p$ ' que l'on associe, dans un système de valeurs communément admis, au contenu propositionnel $p: p$ (être moche) implique $p^{\prime}=$ non $r$ (ne pas avoir du succès auprès des femmes) et vice versa $r$ implique $r$ ' non $p$. Les conclusions que l'on est amené à tirer respectivement de $p$ et de $r$ sont contraires, elles aussi. La structure pourrait être schématisée comme suit :

Type 1a

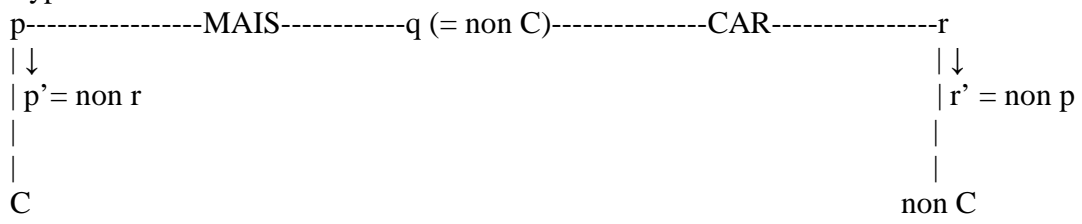

Dans ce type de structure, il serait possible de mettre un POURTANT dans la proposition $r$ :

«X est moche, mais il faut l'envoyer à la mission, car il a pourtant du succès auprès des femmes !»

Dans le second cas les implications $p$ ', $r$ ' que l'on peut tirer des contenus propositionnels ne sont pas contraires aux $r, p$ respectivement - dans un système de valeurs communément admis, il n'y a rien d'antinomique entre le fait d'être moche et de savoir parler chinois. L'opposition se situe uniquement au niveau des conclusions. On peut le schématiser ainsi :

Type $1 b$

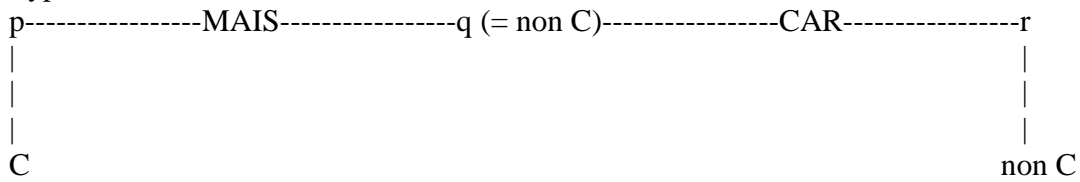

Mettre un POURTANT dans la proposition $r$ serait dans ce cas très problématique :

?? «X est moche, mais il faut l'envoyer à la mission, car il parle pourtant très bien chinois ! »

\footnotetext{
${ }^{7}$ Pour désigner l'énoncé $p$ ' nous préférons utiliser le terme «implication » (qui n'est pas employé ici dans sons sens strictement logique) plutôt que celui de «conclusion » (comme le fait par exemple O. Ducrot ou J.-M. Adam), que nous réservons pour l'énoncé «il faut envoyer à la mission », afin de ne pas confondre les deux.
} 


\subsection{Deuxième type}

Le deuxième type de la structure peut être observé dans le dialogue suivant:

Conseiller $1:$ : $\mathrm{X}$ est moche !»

Conseiller 2 : «X est moche, mais il a du succès auprès des femmes, car il est éloquent!»

Conseiller $3:$ «X est moche, mais il parle très bien chinois, car il est très doué pour les langues !»

Nous voyons qu'à la place des propositions $q$ se trouvent ici les mêmes propositions qui fonctionnaient comme $r$ dans le cas précédent. La conclusion vers laquelle le locuteur (les Conseillers 2 et 3) oriente sont argumentation est toujours la même que dans le cas précédent (c'est-à-dire « il faut envoyer X à la mission »), sauf qu'ici, elle n'est pas explicitée dans une proposition autonome. La proposition $r$ est ici plus une justification/explication de l'énonciation du $q$ qu'un argument en faveur de la conclusion visée. Ce que nous avons dit plus haut à propos de la relation entre les implications que l'on tire dans un système de valeurs communément admis des contenus propositionnels de $p, r$ vaut ici bien évidemment pour $p, q$, le connecteur POURTANT ne pouvant figurer que dans la première proposition $q$ : «X est moche, mais il a pourtant du succès auprès des femmes $\gg$.

Les deux structures peuvent être représentées comme suit :

Type $2 \mathrm{a}$

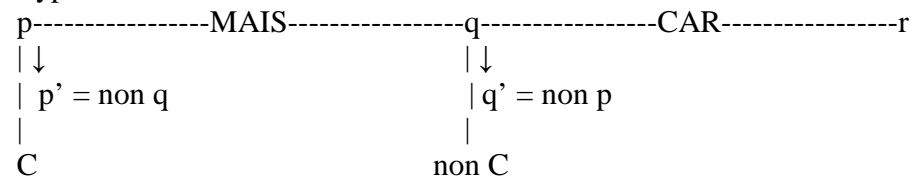

Type $2 b$

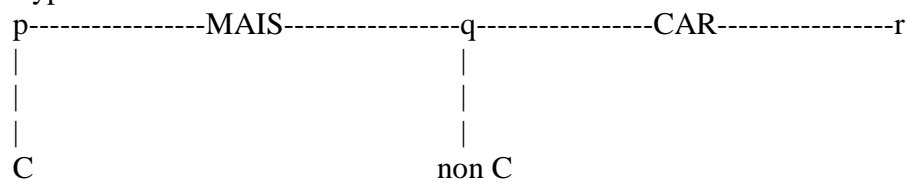

Il est intéressant de faire remarquer que si c'est le premier type de la structure $p$ [MAIS q CAR $r$ ] qui est actualisé, la proposition $r$ est habituellement fournie dans le discours ; son absence, si elle n'est pas justifiée par le co(n)texte, mène aux questions du type : «Et pourquoi êtes-vous de cet avis ? ». Considérons dans notre situation de communication concrète définie plus haut l'énoncé suivant : «X est moche, mais il faut l'envoyer à la mission !».

Dans le deuxième type, la proposition $r$ est largement facultative, quelque peu secondaire par rapport au $q$ qu'elle vient consolider. Son absence n'a généralement pas d'impact sur les effets pragmatiques de l'argumentation.

\footnotetext{
${ }^{8}$ Mettre un POURTANT dans la deuxième proposition q serait problématique : ?? «X est moche, mais il parle pourtant très bien chinois ». En considérant ces différences, J.-M. ADAM (1990 : 192221) établit une distinction entre MAIS concessif et MAIS argumentatif.
} 
Dans les deux cas de figure, la proposition $C A R r$ est intimement liée à l'argumentation. Soit elle apporte l'argument fort pour la conclusion non $C$ exprimée par $q$, soit elle corrobore l'argument fort $q$ en apportant des éléments de justification.

Nous croyons donc pouvoir affirmer une fois de plus que les propositionsénoncés $C A R r$ des structures $p$ [MAIS $q C A R r$ ] qui, apportant une justification à l'argumentation $p$ MAIS $q$, font partie des séquences/périodes argumentatives dans le cadre desquelles elles apparaissent.

En appliquant cette typologie à l'extrait du Roman de la Rose, nous pouvons dire que les structures $p$ [MAIS $q C A R r$ qui y sont actualisées à trois reprises relèvent toutes du type $2 \mathrm{a}$ de notre classement.

\section{Le schéma de la séquence}

Nous revenons maintenant à la question que nous nous sommes posée plus haut, celle de savoir de quel type de structure séquentielle il s'agit dans notre extrait. Étant donné ce qui a été dit au sujet des propositions-énoncés $C A R r$ des structures $p$ [MAIS q CAR r], nous pouvons conclure que le texte analysé est homogène du point de vue séquentiel, car il comporte une séquence argumentative épaulée de deux justifications qui en font partie. La deuxième justification introduite par $\mathrm{CAR}_{2}$ englobe une période argumentative qui inclut elle-même une justification (structure $p$ [MAIS $q$ CAR $r]$ ). Nous pouvons le schématiser comme suit :

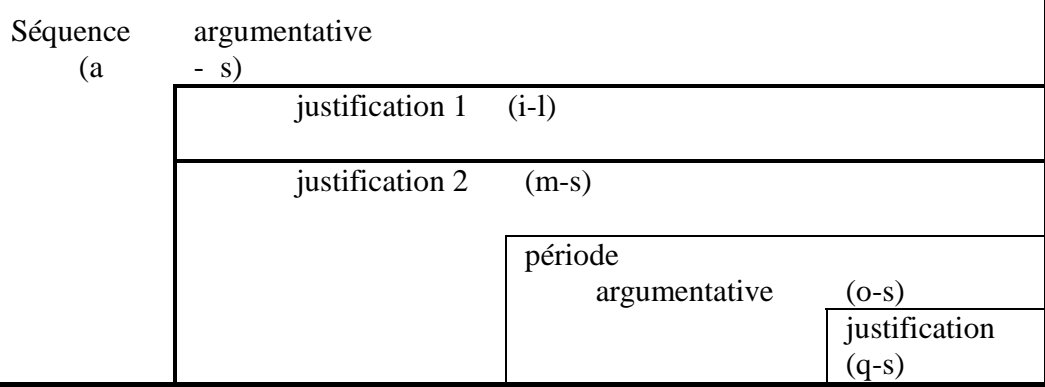

\section{Les connecteurs et leur portée dans la structure textuelle}

6.1. Le fonctionnement argumentatif de chacun des connecteurs argumentatifs de notre passage a été analysé en détail plus haut. Nous allons dire maintenant quelques mots à propos du rôle qu'ont ces connecteurs pour la structuration textuelle. En segmentant et regroupant des propositions, ils contrôlent des portions de texte qui se trouvent sous leur portée et qui peuvent être d'une longueur et d'une complexité variable. Ils délimitent et hiérarchisent ainsi des entités textuelles (séquences, périodes, propositions-énoncés) en leur affectant de plus une orientation argumentative dont ils sont porteurs ${ }^{9}$. Le récepteur du texte est ensuite invité à traiter ces entités globalement comme des éléments constitutifs de la

\footnotetext{
${ }^{9}$ Seuls les connecteurs argumentatifs assurent à la fois la structuration textuelle, la prise en charge énonciative et l'orientation argumentative (cf. ADAM, 2005 : 117)
} 
structure sémantique du texte selon les instructions que lui donnent les connecteurs.

La portée des connecteurs de notre extrait et leur rôle dans la segmentation et dans la hiérarchisation des entités textuelles pourraient être représentés comme suit ( les signes $<>$ signalent la portée du connecteur respectivement à gauche et à droite)

$(\mathrm{a}, \mathrm{b}, \mathrm{c}, \mathrm{d})<\mathrm{MAIS}_{1}>(\mathrm{e}, \mathrm{f}, \mathrm{g}, \mathrm{h})$

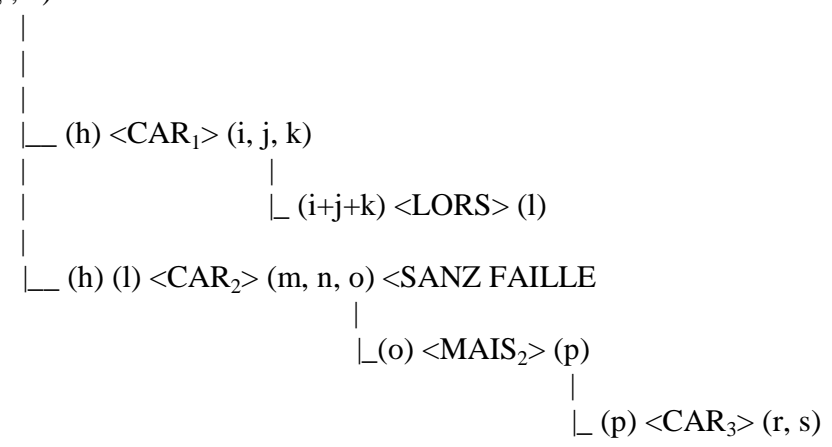

Ainsi apparaît-il clairement que le segment placé hiérarchiquement le plus haut est celui articulé par MAIS ${ }_{1}$ (le noyau de la séquence argumentative), ce segment «domine» deux segments de rang équivalent délimités par $\mathrm{CAR}_{1}$ et $\mathrm{CAR}_{2}$ auxquels le connecteur affecte la valeur de justification. Les deux segments et celui introduit par $\mathrm{CAR}_{2}$ en particulier sont segmentés à leur tour (période argumentative + justification) en entités hiérarchiquement inférieures et donc dominées par l'orientation argumentative (justification) que donnent aux ensembles des propositions (i-1) et (m-s) les connecteurs $\mathrm{CAR}_{1}$ et $\mathrm{CAR}_{2}$.

\subsection{Le MAIS de transition}

La capacité qu'ont les connecteurs argumentatifs à structurer le texte se manifeste d'une manière édifiante dans les vers 17105 et sqq qui suivent directement notre passage :

MAIS de soudre la question / Comment predestinacion / Et la devine presciance, / Plaine de toute porveance, / Puet estre o volenté delivre, / Forz est a gens lais a descrivre.

Ces vers sont introduits par un MAIS dont le fonctionnement est particulièrement intéressant: il s'agit en effet d'un MAIS de transition dont les particularités ont été décrites par A. RoDRIGUEZ-SOMOLINOS (2002: 523-524). L'emploi de ce connecteur permet au locuteur d'établir le lien avec ce qui précède tout en réorientant le discours vers une autre thématique. La proposition qui suit ce MAIS justifie en quelque sorte la continuation du texte. Dans le passage concret que nous sommes en train d'analyser, l'instruction que donne ce MAIS au lecteur peut être paraphrasée comme suit : « de ce qui précède vous pouvez être amenés à conclure que la question du prédéterminisme que nous venons d'exposer est facile et résolue et que notre texte peut prendre fin, or il ne faut pas, car cette question présente d'autres aspects polémiques - le texte doit continuer ». L'argument $p$ de ce MAIS ( $p$ MAIS $q$ ) est constitué par la séquence précédente tout entière. En assurant la transition thématique, le MAIS du vers 17105 marque clairement la fin d'une séquence et le début d'une autre. Que cette transition soit soutenue par le 
mouvement argumentatif propre au connecteur MAIS est ici quelque peu secondaire par rapport au rôle que ce MAIS joue dans la structuration du texte.

\section{Les marqueurs de prise en charge énonciative}

Les marqueurs de prise en charge énonciative ont aussi chacun une portée, comme les connecteurs. A la différence de ces derniers, ils ne participent pas à la structuration du texte et ne sont pas porteurs d'une orientation argumentative. Leur rôle est d'attribuer les portions de textes qu'ils contrôlent à une source énonciative responsable d'un point de vue $(\mathrm{PdV})$, de situer les ensembles de propositions sous leur portée dans des univers de croyance. Ils signalent ainsi quel énonciateur est le garant de la vérité des propos avancés et dans le cas où plusieurs instances énonciatives peuvent être identifiées, ils désignent ces différentes sources de la polyphonie. Dans notre extrait, la portée des différents marqueurs de prise en charge énonciative peut être schématisée comme suit :

[Si dit on> (b, c, d)] [je sai> (f, g, h, i, j, k, 1, m, n, p) [bien sait chascuns sages hom $>(\mathrm{r}, \mathrm{s})]]$

On voit bien, que les énoncés $(b+c+d)$ sont pris en charge par un énonciateur E2 dont le point de vue est présenté comme dissocié de celui du locuteur. En revanche, les énoncés qui suivent MAIS $_{1}$ sont pris en charge par le locuteur (qui adhère au point de vue d'un énonciateur E1). On pourrait le schématiser comme suit :

PdV2 $\quad \mathrm{E} 2 \neq \mathrm{L} \quad$ Si dit on $(\mathrm{b}+\mathrm{c}+\mathrm{d})$

MAIS $_{1}$

PdV1 E1=L je sai $(\mathrm{e}+\mathrm{f}+\ldots . . \mathrm{s})$

Quoique ces marqueurs n'explicitent pas l'orientation argumentative des énoncés, la prise en charge énonciative est inséparable de l'argumentation. La formule «On dit que D0 donc C0, MAIS je sais que même si D0 dans une grande mesure, pas nécessairement $\mathrm{C} 0$ », que nous avons analysée plus haut, ne réalise sa fonction argumentative que grâce aux marqueurs de prise en charge énonciative : leur absence mènerait à un énoncé contradictoire, dont la réalisation en discours serait difficilement imaginable. Considérons à titre d'exemple cet énoncé fabriqué : «?? Il fait beau, donc il faut sortir, mais même s'il fait très beau, il ne faut pas nécessairement sortir. »

\section{Conclusion}

Notre analyse nous a permis de démontrer la pertinence de la notion de prototype pour l'analyse textuelle. L'agencement des entités textuelles concrètes présente un degré variable de typicalité, mais on peut en rendre compte d'une manière unifiée en rattachant ces entités aux modèles prototypiques des séquences textuelles. Les meilleurs représentants de leurs catégories servent ainsi de référence pour la description d'une unité textuelle concrète.

Le passage que nous avons analysé comportait un certain nombre de structures syntaxiques spécifiques dont le fonctionnement textuel et argumentatif était particulièrement intéressant. En partant des occurrences concrètes, nous avons 
proposé une description générale, applicable, croyons-nous, à toute actualisation de ce type de structure.

Si nous avons insisté dans la dernière partie de notre article sur le rôle que jouent les connecteurs pour la structuration du texte, c'est que nous voulions souligner l'importance de cette propriété constitutive du fonctionnement des connecteurs argumentatifs. Aussi sommes-nous persuadé que toute description complexe et rigoureuse de ces unités devrait en tenir compte.

\title{
BIBLIOGRAPHIE
}

Le Roman de la Rose ; édition Strubel, Armand (1992), Paris, Livre de Poche, Lettres gothiques.

ADAM, J.-M. (1990), Éléments de linguistique textuelle, Liège, Mardaga.

ADAM, J.-M. (1994), Les textes : types et prototypes, Paris, Nathan.

ADAM, J.-M. (1999), Linguistique textuelle: des genres de discours aux textes, Paris, Nathan/HER.

ADAM, J.-M. (2005), La linguistique textuelle. Introduction à l'analyse textuelle des discours. Paris, Armand Colin.

DELBEY, A. (1988), Les connecteurs car - que - puisque et la justification en ancien français, Revue de linguistique romane 52, p. 397-419.

DUCROT, O. et al. (1980), Les mots du discours, Paris, Les éditions de minuit.

Groupe $\lambda$-1 (1975), Car, parce que, puisque, Revue Romane 10, p. 248-280.

MoEschler, J. (1985), Argumentation et Conversation, Paris, Hatier.

RodRIGUEZ-SOMOLINOS, A (2002), Ainz et mais en ancien français, Romania vol. 120 (3-4), p. 505-541.

SoUTET, O. (1992), Études d'ancien et de moyen français, Paris, PUF.

\begin{abstract}
The concept of prototype is very operational for the textual analysis of discourse - the concrete occurrences of textual units can be successfully described in reference to the prototypical scheme of a textual sequence even if the degree of typicality of the unit being described is quite low. The syntactic structures of the short passage of the Romance of the Rose by Jean de Meun we studied in this paper have a specific and interesting function in argumentation, their pragmatic role appear clearly when this structures are analyzed as a part of a textual sequence. Our analysis pointed out that the argumentative function of connectors is inseparable of the textual one.
\end{abstract}

Le présent article a été rédigé grâce au soutien financier attribué par la Grantová agentura České republiky dans le cadre du projet GAČR 405/08/P101. 